\title{
Attenuation of post-myocardial infarction depression in rats by $n-3$ fatty acids or probiotics starting after the onset of reperfusion
}

\author{
Kim Gilbert $^{1,2}$, Jessica Arseneault-Bréard ${ }^{1,2}$, Fabio Flores Monaco ${ }^{1}$, Alexanne Beaudoin ${ }^{1}$, \\ Thierno Madjou Bah ${ }^{1,3}$, Thomas A. Tompkins ${ }^{4}$, Roger Godbout ${ }^{1,3}$ and Guy Rousseau ${ }^{1,2_{*}}$ \\ ${ }^{1}$ Centre de biomédecine, Hôpital du Sacré-Cour de Montréal, 5400 Boulevard Gouin Ouest, Montréal, Québec, \\ Canada H4J $1 C 5$ \\ ${ }^{2}$ Département de pharmacologie, Université de Montréal, CP Centre-Ville, Montréal, Québec, Canada H3C3J7 \\ ${ }^{3}$ Département de psychiatrie, Université de Montréal, CP Centre-Ville, Montréal, Québec, Canada H3C3J7 \\ ${ }^{4}$ Institut Rosell-Lallemand Inc., 6100 Royalmount, Montréal, Québec, Canada H4P 2 R2
}

(Submitted 30 March 2012 - Final revision received 26 July 2012 - Accepted 26 July 2012 - First published online 15 October 2012)

\begin{abstract}
Proinflammatory cytokines play a central role in depression-like behaviour and apoptosis in the limbic system after myocardial infarction (MI). A PUFA $n-3$ diet or the combination of Lactobacillus helveticus R0052 and Bifidobacterium longum R0175 probiotics, when given before the ischaemic period, reduce circulating proinflammatory cytokines as well as apoptosis in the limbic system. The present study was designed to determine if the same nutritional interventions maintain their beneficial effects when started after the onset of the reperfusion period and attenuate depression-like behaviour observed after MI. MI was induced by the occlusion of the left anterior descending coronary artery for $40 \mathrm{~min}$ in rats. After the onset of reperfusion, animals were fed with a high- or low-PUFA $n$ - 3 diet, combined or not with one billion live bacteria of $L$. helveticus and B. longum. At $3 \mathrm{~d}$ post-MI, caspase-3 enzymatic activities and terminal $2^{\prime}$-deoxyuridine, $5^{\prime}$-triphosphate (dUTP) nick-end labelling (TUNEL)-positive cells were decreased in the CA1, dentate gyrus (DG) and amygdala with the high-PUFA $n$ - 3 diet, as compared to the three other diets. Probiotics attenuated caspase- 3 activity and TUNEL-positive cells in the DG and the medial amygdala. At 2 weeks post-MI, depression-like behaviour was observed in the low-PUFA $n-3$ diet without probiotics-group, and this behaviour was attenuated with the high-PUFA $n-3$ diet or/and probiotics. These results indicate that a high-PUFA $n-3$ diet or the administration of probiotics, starting after the onset of reperfusion, are beneficial to attenuate apoptosis in the limbic system and post-MI depression in the rat.
\end{abstract}

Key words: n-3 Fatty acids: Apoptosis: Limbic system: Diet: Behaviour: Hippocampus: Amygdala

Depressive mood is common after myocardial infarction $(\mathrm{MI})^{(1)}$ and $20 \%$ of MI patients develop an episode of major depression $^{(2)}$. The association of depression with MI is critical, as the mortality rate is increased three to four times in depressed MI patients compared to non-depressed MI patients ${ }^{(3)}$. As the level of non-responders is still high with antidepressant treatments ${ }^{(4,5)}$ and that the treatment may interfere with myocardial infarct size ${ }^{(6)}$, new antidepressant strategies need to be identified for such cases.

The physiopathology of depression is complex and different mechanisms are involved ${ }^{(7)}$. Our previous work and that of others indicate that proinflammatory cytokines may play a key role in depression-like behaviour ${ }^{(8-10)}$. Indeed, injection of proinflammatory cytokines is followed by depression-like behaviour $^{(11)}$. Post-MI increased levels of proinflammatory cytokines are accompanied by apoptosis in the hippocampus and the amygdala, another physiopathological element of depression $^{(8,9,12)}$.

Dietary interventions can be used to prevent the post-MI elevation of proinflammatory cytokines. For example, we have shown that high-PUFA $n-3$ diets reduce the circulating level of TNF $\alpha$ after $\mathrm{MI}^{(13)}$. High-PUFA $n$-3 diets also reduce levels of monocyte chemotactic protein-1 (MCP-1), a chemokine that contributes to inflammatory states ${ }^{(14,15)}$. In parallel, it has been observed that Lactobacillus belveticus R0052 reduces proinflammatory cytokines IL- $1 \beta$ and IL- 6 , but nonsignificantly reduces $\mathrm{TNF}-\alpha^{(16)}$, whereas Bifidobacterium longum $\mathrm{R} 0175$ can reduce TNF- $\alpha$ as well as IL-8 ${ }^{(17)}$. Combined

Abbreviations: AR, area at risk; CA1, hippocampal CA1; CA3, hippocampal CA3; DG, dentate gyrus; LA, lateral amygdala; MA, medial amygdala; MCP-1, monocyte chemotactic protein-1; MI, myocardial infarction; TUNEL, terminal dUTP nick-end labelling. 
with L. helveticus R0175, B. longum subsp. infantis R0033 and $B$. bifidum R0071 can also elevate the concentrations of anti-inflammatory cytokines IL- ${ }^{(16)}$, modifying the balance between pro- and anti-inflammatory cytokines. The fact that such nutritional interventions also reduce apoptosis in the limbic system suggests a potential antidepressant-like effect. The objective of the present study was thus to determine if probiotics and $n$-3-rich diets, combined or alone, could be beneficial to attenuate post-MI depression-like behaviour. While previous studies ${ }^{(13,18)}$ used nutritional strategies before heart ischaemia, the present protocol aimed at implementing the nutritional intervention after $\mathrm{MI}$ in order to increase clinical relevance.

\section{Experimental methods}

\section{Ethics statement}

These experiments conformed to animal care guidelines published by the Canadian Council on Animal Care, and the procedures performed were approved by the local Animal Care Committee of Hôpital du Sacré-Coeur de Montréal.

\section{Experimental design}

We studied a total of sixty-four male, 3-month-old SpragueDawley rats weighing 325-350g at the start of the experiments (Charles River Canada). Rats were housed individually under constant conditions (temperature of $21-22^{\circ} \mathrm{C}$ and humidity of $40-50 \%$ ), including a $12 \mathrm{~h}$ dark-12 h light cycle, which began at 08.00 hours. Chow pellets and tap water were available ad libitum throughout the study. An acclimatisation period of $3 \mathrm{~d}$ after delivery by the supplier was allowed before the rats were randomly distributed in one of the four groups: a low- or high- $n$-3 PUFA diet in combination or not with probiotics L. helveticus R0052 and B. longum R0175 (mentioned later). Six rats from each group (a total of twenty-four) were killed $72 \mathrm{~h}$ post-MI for biochemical analyses and ten rats from each group (a total of forty) were killed $18 \mathrm{~d}$ post-MI after behavioural tests.

\section{Diets}

Chow pellets rich in lipid (22\% protein, $42.9 \%$ carbohydrate, $20 \cdot 3 \%$ fat, by weight) were supplied by Harlan Teklad. The respective composition in fatty acids is presented in Table 1.

\section{Probiotics}

The probiotics that were given were a commercially available combination of two genera: L. helveticus R0052 and B. longum R0175 (Probio'Stick ${ }^{\mathrm{TM}}$ provided by Institut Rosell, Inc.). The probiotics were administered by dissolving the freeze-dried culture or the vehicle only (maltodextrin) in $200 \mathrm{ml}$ of drinking tap water. Each rat in the probiotics group received a daily dose of $10^{9}$ colony-forming units. The drinking solution was newly prepared every 2 nd day for the duration of experiments. Water intake was monitored throughout the entire investigation
Table 1. Composition of PUFA in the different diets $(\mathrm{g} / \mathrm{kg})$

\begin{tabular}{lrr}
\hline & \multicolumn{2}{c}{ Diets } \\
\cline { 2 - 3 } & High & Low \\
\hline Total fat & $202 \cdot 5$ & $202 \cdot 5$ \\
Saturated fat & $78 \cdot 1$ & $71 \cdot 6$ \\
Monounsaturated fat & $33 \cdot 7$ & $34 \cdot 8$ \\
Polyunsaturated fat & $83 \cdot 2$ & $92 \cdot 5$ \\
$n-6$ PUFA & & \\
$18: 2 n-6$ (LA) & $38 \cdot 9$ & $76 \cdot 7$ \\
$20: 4 n-6$ (AA) & $1 \cdot 3$ & 0 \\
$n-3$ PUFA & & \\
$18: 3 n-3$ (ALA) & 22 & $15 \cdot 6$ \\
$20: 5 n-3$ (EPA) & $9 \cdot 3$ & 0 \\
$22: 5 n-3$ (DPA) & $2 \cdot 3$ & 0 \\
$22: 6 n-3$ (DHA) & $6 \cdot 3$ & 0 \\
\hline LA, linoleic acid; AA, arachidonic acid; & ALA, \\
$\alpha$-linolenic acid; DPA, docosapentaenoic acid.
\end{tabular}

to ensure that sufficient bacteria were administered. The body weight of each rat was also monitored every day.

\section{Surgical procedure}

Animals were anaesthetised following a ketamine/xylazine (50 and $5 \mathrm{mg} / \mathrm{kg}$, respectively) intramuscular injection. Subsequently, the rats were intubated and anaesthesia was maintained using isoflurane (1.2\%) ventilation. Electrocardiogram and heart rate of the rats were monitored throughout the procedure using electrodes placed on their paws. A left thoracotomy was carried out at the fifth intercostal space, permitting the occlusion of the left anterior descending coronary artery using a 4-0 silk suture (Syneture; Covidien) and plastic snare. Ischaemia was confirmed by alterations of the ST segment and the presence of the ventricular subepicardial cyanosis. After $40 \mathrm{~min}$ of ischaemia, the thread was removed, permitting reperfusion of the myocardial tissue. Then, the thorax was sutured by means of a 2-0 silk suture (Syneture; Covidien), and the animals were given an antibiotic intramuscular injection (15000 IU penicillin G; Duplocillin LA, Intervet Canada Limited) as well as an analgesic subcutaneous injection ( $2 \mathrm{mg} / \mathrm{kg}$ buprenorphine) before being returned to their respective cages. The animals received a second analgesic injection $(0.05 \mathrm{mg} / \mathrm{kg}$ buprenorphine $)$ the day after surgery.

Decapitation measurements, the area at risk of the heart, myocardial infarct size and brain dissection

After the reperfusion period ( 3 or $18 \mathrm{~d}$ ), the animals were restrained in a cone bag and rapidly killed by decapitation, a method that avoids alterations of biochemical pathways that could arise ensuing anaesthesia or $\mathrm{CO}_{2}$ exposure. Blood samples were taken from the arteries after the decapitation. The brains and hearts were rapidly removed and placed in a dish kept on crushed ice. The dentate gyrus (DG), CA1 and CA3 areas of the hippocampus as well as the lateral amygdala (LA) and medial amygdala (MA) were excised from the brains, snap-frozen in liquid $\mathrm{N}_{2}$ and stored at $-80^{\circ} \mathrm{C}$ until needed. 
The hearts were removed and washed with saline by retrograde perfusion into aorta. The left anterior descending coronary artery was occluded at the same site as for inducing MI (as mentioned earlier) to map the area at risk (AR) using an infusion of Evans blue (0.5\%). The hearts were frozen $\left(-80^{\circ} \mathrm{C}\right.$ for $5 \mathrm{~min}$ ), sliced into four transverse sections of $2 \mathrm{~mm}$ and placed in 2,3,5-triphenyltetrazolium $(1 \%, \mathrm{pH} 7.4)$ at $37^{\circ} \mathrm{C}$ for 10 min to better distinguish the area of necrosis (I) from AR. The MI was expressed as a percentage of necrosis (I) of the $\mathrm{AR}((\mathrm{I} / \mathrm{AR}) \times 100)$. Additionally, AR was expressed as a percentage of left ventricle area $(\mathrm{LV})((\mathrm{AR} / \mathrm{LV}) \times 100)$.

\section{Biochemical analysis}

Caspase-3 activity. Caspase-3 activity was measured according to the protocol described previously ${ }^{(19)}$. Tissues were homogenised by sonification in lysis buffer and incubated for $30 \mathrm{~min}$ on ice. The tissue homogenates were centrifuged at $4^{\circ} \mathrm{C}$ for $10 \mathrm{~min}$. Enzymatic reactions were undertaken in reaction buffer with $25 \mathrm{mg}$ of protein (attested by the Bradford method) and fluorescent substrate (Ac-DEVD-AMC; CEDARLANE; $40 \mu \mathrm{M}$ ). Reactions were studied after incubation in the dark for $3 \mathrm{~h}$ at $37^{\circ} \mathrm{C}$, and stopped with the addition of $0.4 \mathrm{M}-\mathrm{NaOH}$ and $0.4 \mathrm{M}$-glycine buffer. Fluorescence was quantified by spectrofluorometry (Photon Technology International) at an excitation wavelength of $365 \mathrm{~nm}$ and emission wavelength of $465 \mathrm{~nm}$.

\section{Terminal dUTP nick-end labelling-positive cells}

TUNEL analysis was undertaken according to the protocol provided by the manufacturer (Oncogene Science, Inc.) and according to the TUNEL protocol described previously ${ }^{(8)}$. In summary, cryostat sections were fixed in paraformaldehyde ( $4 \%$ ) and rinsed, then permeabilised and, after washing, the labelling reaction was performed for $1 \mathrm{~h}$ at $37^{\circ} \mathrm{C}$. The reaction was stopped, and all nuclei were stained with propidium iodide $(1 \mu \mathrm{m})$. The sample was viewed in a Leica epiillumination microscope with a filter for $4^{\prime}, 6$-diamidino-2phenylindole (DAPI) $(330-380 \mathrm{~nm})$. All sections were photographed at the same magnification $(400 \times)$ with a Nikon Coolpix 995 camera (Nikon Canada). A total of 20-25 pictures were taken of each sample, with quantification of positive cells on total number.

\section{Rat cytokines/chemokines assay}

A cytokine/chemokine assay was performed according to the protocol provided by the manufacturer (Rat Cytokine/Chemokine Magnetic Bead 96-Well Plate, Millipore Canada). In brief, the plasma was diluted by 5 -fold and $200 \mu$ l of assay buffer was added into each well of the plate, mixed for $10 \mathrm{~min}$ at room temperature and decanted. The diluted samples were incubated overnight at $4^{\circ} \mathrm{C}$ in the reaction mix in the presence of beads, and the next morning, the plates were washed and incubated for $2 \mathrm{~h}$ at room temperature with detection antibodies. After that, streptavidin-phycoerythrin solution was added to the wells for $30 \mathrm{~min}$ followed by the addition of sheath fluid. Plates were run on a Bio-Plex 200 system with high-throughout fluidics (Bio-Rad). The median fluorescent intensity was analysed to determine the plasma concentrations of MCP-1 and IL-4.

\section{Behavioural measures}

The tests employed were selected on the basis of their validity regarding behavioural depression syndrome. All tests were conducted individually, in the morning, starting $12 \mathrm{~d}$ after surgery. Social interaction was evaluated on day 12 , the forced swimming test was performed on days 15 and 16 and the passive avoidance step-down test on day 17. All animals were killed on day 18 .

\section{Social interaction test}

Pairs of rats were each placed in a clean shoebox for $10 \mathrm{~min}$. During this period, two observers without knowledge of the experimental condition observed one animal each; the duration and number of interactions with the other rat was measured. Rats were tested between 09.00 and 11.00 hours.

\section{Forced swim test}

Rats were placed individually in a transparent $25 \mathrm{~cm}$ diameter pool filled to a depth of $30 \mathrm{~cm}$ with $22-25^{\circ} \mathrm{C}$ water, with no possible escape. Two observers without knowledge of the experimental condition used identical chronometers to time the immobile, swim and escape trial periods of the animals. The test was conducted for $2 \mathrm{~d}$ : day 1 comprised $15 \mathrm{~min}$ of habituation and day 2 entailed the actual 5 min test. On the 2nd day, immobility time was translated into depressive symptoms by comparison to the controls. Rats were tested between 09.00 and 11.00 hours.

\section{Passive avoidance step-down test}

Rats were placed individually in a test chamber $(14 \mathrm{~cm} \times 23 \mathrm{~cm})$ on a Plexiglas platform $(14 \mathrm{~cm} \times 19 \mathrm{~cm})$. An electrifiable grid $(14 \times 14 \mathrm{~cm})$ is placed alongside and $2.5 \mathrm{~cm}$ lower than the Plexiglas platform $(14 \mathrm{~cm} \times 14 \mathrm{~cm})$. When the

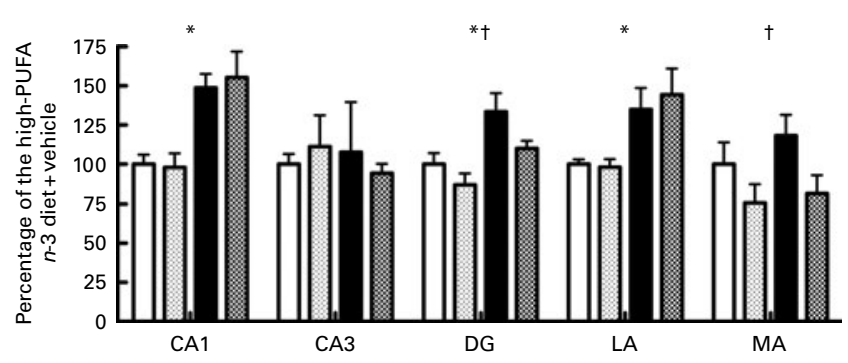

Fig. 1. Caspase-3 activity, expressed as a percentage of the high- $n-3$ PUFA diet + vehicle, in different limbic regions assessed by in vitro spectrofluorescence after $72 \mathrm{~h}$ of reperfusion. CA1, hippocampal CA1; CA3, hippocampal CA3, DG, dentate gyrus; LA, lateral amygdala; MA, medial amygdala. Values are means, with their standard errors represented by vertical bars (n 4-6 per group). * Mean values were significantly different for the diet factor $(P<0.05)$. † Mean values were significantly different for the probiotic factor $(P<0.05)$. $\sqsubset$, High-PUFA $n-3+$ vehicle; $\leftleftarrows$, high-PUFA $n-3+$ probiotics; 口, low-PUFA $n-3+$ vehicle; ⿷匚⿱ , low-PUFA $n-3+$ probiotics. 
Table 2. Percentage of terminal dUTP nick-end labelling-positive cells on total cell count in different limbic regions analysed at $72 \mathrm{~h}$ after reperfusion

\begin{tabular}{|c|c|c|c|c|c|c|}
\hline & \multicolumn{2}{|c|}{ High-PUFA $n-3$ diet } & \multicolumn{2}{|c|}{ Low-PUFA $n$-3 diet } & \multicolumn{2}{|c|}{$P$} \\
\hline & Without probiotics & With probiotics & Without probiotics & With probiotics & Diets & Probiotics \\
\hline $\mathrm{CA} 1$ & 1.40 & 1.23 & 2.52 & 1.84 & $<0.05$ & NS \\
\hline CA3 & 0.58 & 0.26 & 0.38 & 0.36 & NS & NS \\
\hline DG & 1.45 & 1.05 & 2.74 & 1.44 & $<0.05$ & $<0.05$ \\
\hline LA & 0.98 & 0.92 & $2 \cdot 74$ & $2 \cdot 28$ & $<0.05$ & NS \\
\hline MA & 1.87 & $1 \cdot 11$ & $2 \cdot 19$ & 1.43 & $<0.05$ & $<0.05$ \\
\hline
\end{tabular}

CA1, hippocampal CA1; CA3, hippocampal CA3; DG, dentate gyrus; LA, lateral amygdala; MA, medial amygdala

animal places all four feet on the electrifiable grid, it receives a mild, brief shock ( $5 \mathrm{~mA}$ for $1 \mathrm{~s}$ ) and is removed from the test chamber. After $30 \mathrm{~s}$, it is placed anew on the platform. If the rat remains on the platform without going onto the grid for $1 \mathrm{~min}$, it is removed from the test box for $30 \mathrm{~s}$. Criterion is reached when the rat avoids going onto the grid for three consecutive trials. The number of trials needed to reach the test criterion and the time needed to learn the test were noted. Rats were tested $1 \mathrm{~d}$ before they were killed.

\section{Statistical analysis}

Data are reported as means with their standard errors. Groups were compared with a two-way factorial ANOVA, using diet and probiotics as factors. If an interaction between diet and probiotics was significant, simple effects were analysed according to Field ${ }^{(20)}$, otherwise if an interaction was not significant, main effects were reported. The MI size and AR were compared using Student's $t$ tests. Number of trials in the passive avoidance test were analysed with the Kruskal-Wallis test $(P<0.05)$ followed by a Mann-Whitney test using Bonferroni correction to adjust for the number of comparisons. The TUNEL staining data were evaluated with $\chi^{2}$ tests followed by portioning according to Siegel \& Castellan ${ }^{(21)} . P<0.05$ was considered significant.

\section{Results}

Food, water consumption and weight gain were similar between groups throughout the protocol (data not shown), indicating that diet had no influence on these measures.

\section{Infarct size}

After $72 \mathrm{~h}$ and $18 \mathrm{~d}$ post-MI reperfusion, MI size was similar between groups and represents about $35 \%$ of the AR (data not shown). This indicates that diets had no protective effect on the ischaemic myocardium when started during the reperfusion period.

\section{Apoptosis}

No significant interaction was observed for caspase-3 activity among the five regions tested after $72 \mathrm{~h}$ of reperfusion. Main effect analysis indicated that caspase- 3 activity was significantly higher in the low- $n-3$ PUFA diet compared to the high- $n$-3 PUFA diet (diet factor) in the CA1 $(F(1,16)=24 \cdot 04$; $P<0.05)$, DG $(F(1,19)=11.27 ; \quad P<0.05)$ and in the LA $(F(1,15)=11.77 ; P<0.05)$ (Fig. 1). Caspase-3 activity was similar in the CA3 and MA for the diet factor (Fig. 1). Analyses of the probiotic factor indicated a significant reduction in caspase-3 activity in the DG $(F(1,19)=4.68 ; P<0.05)$ and MA $(F(1,15)=5.63 ; P<0.05)$ areas. No significant difference was observed in the CA1, CA3 and in the LA for the probiotic factor $(P>0.05$; Fig. 1).

The TUNEL-positive cells were significantly increased in the low- $n$-3 PUFA diet compared to the high- $n$-3 PUFA diet in the CA1, DG, LA and MA regions. The probiotics group showed a decrease in the TUNEL-positive cells in the DG and MA regions $v$. the placebo group $(P<0.05$; Table 2$)$. No other difference was observed.

\section{Behavioural tests}

Analyses of social interaction duration disclosed a significant interaction between diet and probiotics $(F(1,28)=5 \cdot 34$; $P<0.05)$. Analysis of simple effects revealed lower values in the low- $n$-3 PUFA diet without probiotics compared to the low- $n$-3 PUFA with probiotics $(F(1,29)=7 \cdot 40 ; \quad P<0.05)$, indicating the beneficial effect of probiotics in the presence of the low- n-3 PUFA diet (Fig. 2).

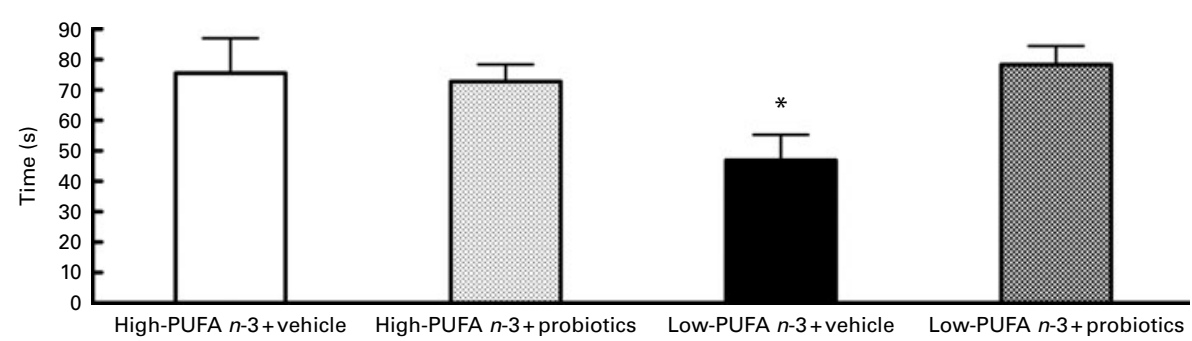

Fig. 2. Social interaction - results of social interaction test proceed on the 12th day of reperfusion in seconds. Values are means, with their standard errors represented by vertical bars ( $n 8-10$ per group). ${ }^{*}$ Indicates a significant interaction $(P<0.05)$. 


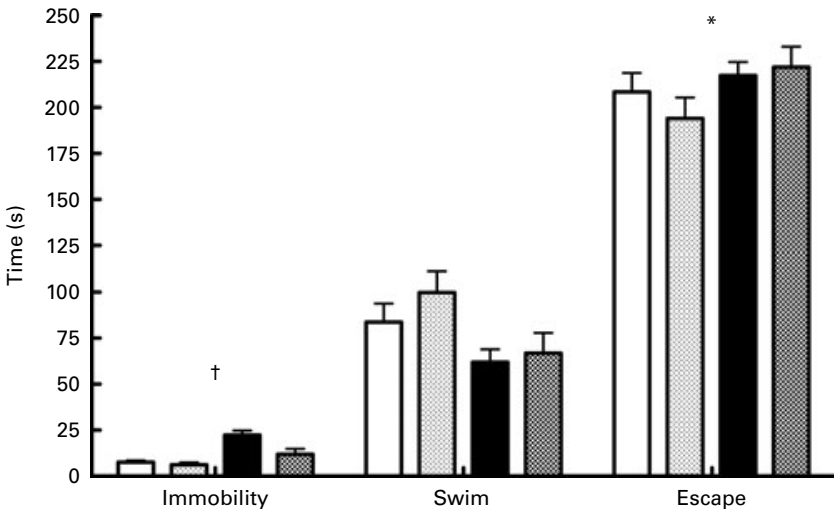

Fig. 3. Forced swim test - results of the forced swim test proceed on the 16th day of reperfusion. Values are means, with their standard errors represented by vertical bars ( $n$ 9-10 per group). * Mean values were significantly different for the diet factor $(P<0.05)$. †Significant interaction for immobility time $(P<0.05)$. ᄃ, High-PUFA $n-3+$ vehicle; $\square$, high-PUFA $n-3+$ probiotics; $\square$, low-PUFA $n-3+$ vehicle; $:$, low-PUFA $n-3+$ probiotics.

Results of the forced swim test (Fig. 3) indicated a significant interaction between diet and probiotics for the immobility variable $(F(1,33)=4.55 ; P<0.05)$. Analysis of simple effects showed that probiotics reduced immobility time in the low$n$-3 PUFA diet $(F(1,34)=7.36 ; P<0.05)$ compared to the low- $n-3$ PUFA diet without probiotics. A significant diet main effect was also found for the escape variable $(F(1,33)=6 \cdot 44$; $P<0.05$ ), indicating a lower escape time in the low- $n-3$ PUFA diet rats. No difference was observed for the swimming time parameter.

The passive avoidance step-down test did not disclose a significant interaction, but the main effect of diet $(F(1,27)=5 \cdot 65$; $P<0.05)$ and probiotics $(F(1,25)=5.33 ; P<0.05)$ was found, indicating that the rats in the high- $n-3$ PUFA diet and the probiotics groups succeeded more rapidly (Fig. 4). Number of trials indicated a significant difference between groups $\left(\chi^{2}=7 \cdot 89, \mathrm{df}=3, P<0.05\right)$. The results indicate that the low- $n-3$ PUFA diet without probiotics group took more attempts to succeed the test, as compared to the low- $n-3$ PUFA diet with probiotics group $(P=0 \cdot 029)$.

\section{Cytokine/chemokine assay}

The probiotic diet was associated with significantly higher IL-4 plasma concentrations compared to vehicle $(F(1,24)=6 \cdot 65$; $P<0.05)$. The low- PUFA $n-3$ diet was associated with significantly higher MCP-1 plasma concentrations compared to the high-PUFA $n$-3 diet $(F(1,30)=4 \cdot 74 ; P<0 \cdot 05$; Fig. 5$)$.

\section{Discussion}

The data obtained in the present study show that a high- $n-3$ PUFA diet implemented for 2 weeks after a MI reduces depression-like behaviour, as documented by three different behavioural tests. Similarly, the combined intake of probiotics L. helveticus R0052 and B. longum R0175 had a positive effect on depression-like behaviour in presence of a low- $n-3$ PUFA diet. The addition of probiotics to the high- $n$ - 3 PUFA diet has no additional effect on behaviour after MI, suggesting a common pathway of action between $n-3$ and probiotics, or alternatively, a plateau effect on behaviour is reached with the high- $n$-3 PUFA diet without any possibility of improvement in the presence of probiotics.

Both nutritional interventions ( $n-3$ and probiotics) were started after the onset of reperfusion and did not have a significant effect on infarct size, as expected. Indeed, cardioprotective interventions need to be started at the onset of reperfusion or soon after to be beneficial for the myocar$\operatorname{dium}^{(22)}$. We have shown, for example, that the administration
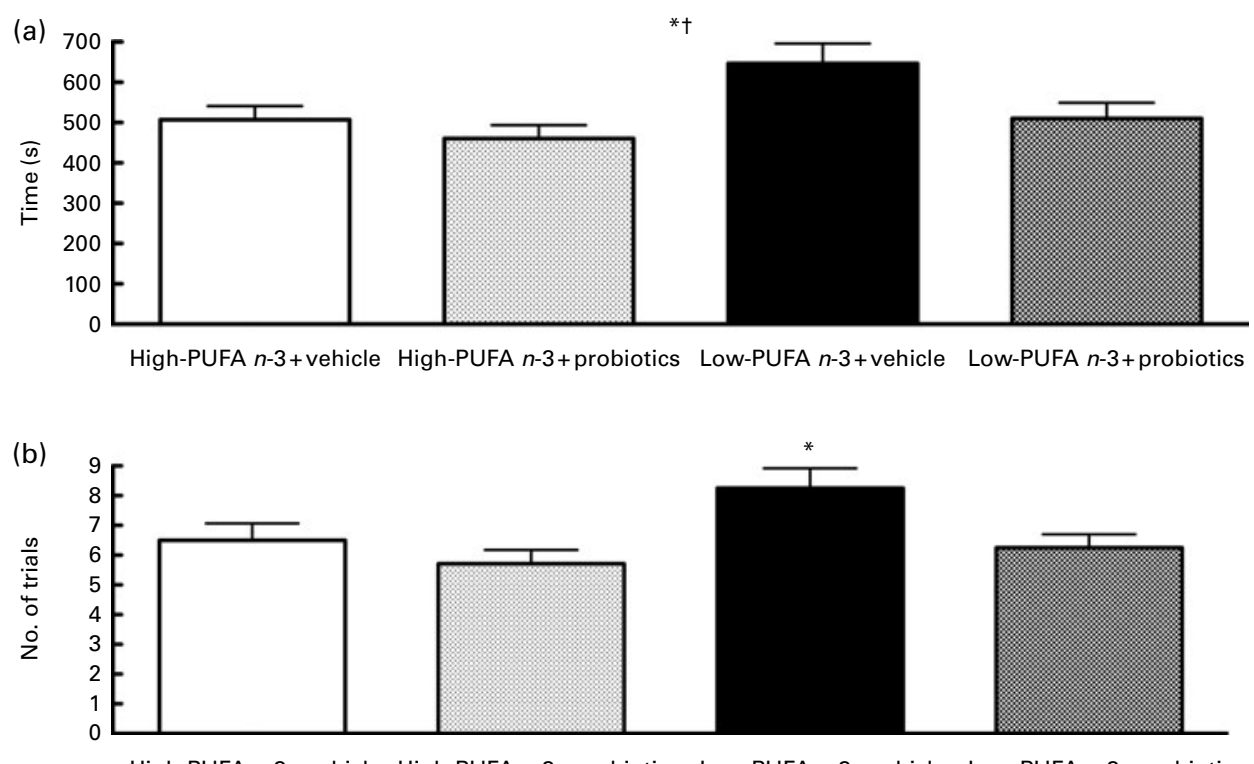

Fig. 4. Passive avoidance test - results of the passive avoidance test proceed on the 17th day of reperfusion. (a) Time to succeed in seconds. Values are means, with their standard errors represented by vertical bars $(n 8-10$ per group). * Mean value was significantly different for the diet factor $(P<0.05)$. $\dagger$ Mean value was significantly different for the probiotic factor $(P<0.05)$. (b) Number of trials. *Value was significantly different between low- $n$-3 PUFA diet + vehicle, as compared to the other groups $(P<0.05)$. 


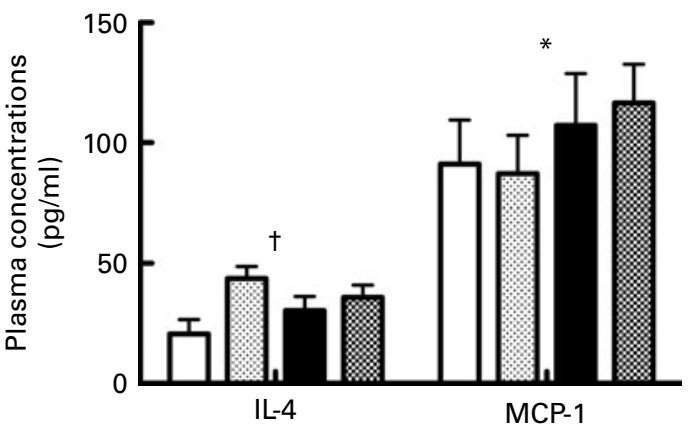

Fig. 5. Plasma concentrations of IL-4 and monocyte chemotactic protein-1 (MCP-1). Values are means, with their standard errors represented by vertical bars ( $n$ 8-10 per group). * Mean value was significantly different for the diet factor $(P<0.05)$. † Mean value was significantly different for the probiotic factor $(P<0.05)$. ᄃ, High-PUFA $n-3+$ vehicle; $匚$, high-PUFA $n-3+$ probiotics; $\square$, low-PUFA $n-3+$ vehicle; ⿷匚 , low-PUFA $n-3+$ probiotics.

of an $\mathrm{A}_{2 \mathrm{~A}}$ adenosine receptor agonist, CGS21680, 5 min before the reperfusion period reduced infarct size, whereas its administration $5 \mathrm{~min}$ after the onset of reperfusion did not ${ }^{(22)}$. In the present study, $n-3$ or probiotics were started after the animals recovered from anaesthesia, i.e. a few hours after the onset of reperfusion.

In previous studies, we documented the presence of apoptosis in the amygdala and hippocampus following MI, with a peak at about the 3 rd day of reperfusion ${ }^{(23,24)}$. Interestingly, pharmacological interventions with antidepressants (sertraline ${ }^{(24)}$ ) or others (probiotics ${ }^{(18)}$ ) have been shown to reduce apoptosis and also to attenuate the depression-like behaviour. The increase in cell death perturbs the homeostasis of the limbic system and can be responsible for the depression-like behaviour. Indeed, it has been shown that neurons that have neither electrical activity nor trophic support die by apoptosis $^{(25)}$ and could explain the development of depression.

We observed that the inhibition of apoptosis by the $n$ - 3 diet or the probiotics is common for the DG and MA, whereas reduction of apoptosis has been observed in the CA1 and LA only in the $n-3$ diet. These results suggest that the DG and MA play a pivotal role in post-MI depression, and their integrity are important to attenuate post-MI depression behaviour.

According to our previous work, proinflammatory cytokines could be partly responsible for apoptosis in the limbic system. In the presence of pentoxifylline ${ }^{(9)}$ or polyethylene glycollinked TNF receptor 1 (PEG-TNFR) ${ }^{(8)}$, apoptosis in the limbic system is attenuated after MI, as compared to untreated animals, suggesting a role of proinflammatory cytokines in apoptosis. The binding of these cytokines, such as TNF $\alpha$, with their receptors activates caspase- 8 and ultimately caspase- 3 .

In the present study, rats treated with probiotics maintain higher concentrations of IL-4, which has been also reported with other probiotics ${ }^{(26,27)}$. IL- 4 is known to modulate microglial activity and can be correlated with a decrease in inflammation $^{(28)}$ and protects host tissues from complement activation $^{(29)}$. Also, it has been reported that probiotics, such as the combination used in the present study, reduce the concentration of proinflammatory cytokines (IL-1 $\beta$ and $\mathrm{TNF} \alpha)^{(16,17)}$. The shift of the pro/anti-inflammatory cytokine balance may contribute to a reduction of apoptosis as well as an improvement of the depression-like behaviour symptoms.

Similarly, we have observed that in presence of the highPUFA $n-3$ diet, the plasma concentrations of MCP-1 were reduced compared to the low- $n-3$ diet. This reduction could be explained by the documented reduction of TNF $\alpha$ which we have previously observed in presence of the same highPUFA $n-3$ diet $^{(13)}$. According to the results obtained by Diaz Encarnacion et al. ${ }^{(14)}$, a high- PUFA $n$ - 3 diet can reduce the TNF $\alpha$-stimulated transcription of the $M C P$ - 1 gene through interaction of signalling pathways involving extracellular signalregulated kinases and NF- $\mathrm{BB}$. A decrease in MCP-1 could be part of the anti-inflammatory and antidepressive action of $n-3$.

The rapidity of the effect of $n-3$ on apoptosis observed in the limbic system may suggest mechanisms other than the incorporation in the membrane. It has been observed that $n$ - 3 metabolites, such as resolvins, may interact with G-proteincoupled receptors such as chemerin receptor $23^{(30)}$ or $G$ protein-coupled receptor $32^{(31)}$ to induce an intracellular signal that could interrupt inflammation by down-regulating $\mathrm{NF}-\kappa \mathrm{B}$ factor, leading to a diminution on inflammatory gene expression such as $\mathrm{TNF}^{(32)}$. The mechanism of this downregulation is not well understood, but it could be due to a decrease of the phosphorylation of inhibitor of NF- $\mathrm{B}$ factor, which inhibits NF- $\mathrm{BB}^{(33)}$. Another potential explanation for the anti-apoptotic properties of $n-3$ PUFA is that DHA is a precursor of neuroprotectin $\mathrm{D}$, a substance that induces pro-survival signals down-regulating apoptosis ${ }^{(34)}$. Systemic DHA administration, after middle cerebral artery occlusion, helps in neuroprotectin D production ${ }^{(35)}$ and may, thus, reduce apoptosis by altering the intrinsic pathway.

The difference between the $n-3$ diets can be explained by the presence of EPA and DHA (long-chain PUFA) in the high- $n-3$ diets and their absence in the low- $n-3$ diet. EPA and DHA are beneficial for the heart and the brain ${ }^{(36)}$ and it has been observed that elongation of $\alpha$-linolenic acid, although limited ${ }^{(37,38)}$, can occur in rats and produce DHA and EPA. In the low- $n-3$ diet, however, $\alpha$-linolenic acid levels may be insufficient or the delay to produce DHA and EPA may be too long to have a positive effect.

\section{Conclusion}

In conclusion, the present data indicate that a high- $n$ - 3 PUFA diet or addition of L. helveticus R0052 and B. longum R0175 to a low- $n$-3 PUFA diet is beneficial after MI to reduce apoptosis in the limbic system and to attenuate depression-like behaviour.

\section{Acknowledgements}

The present work was supported by La Fondation des Maladies du Coeur du Québec. The authors declare no conflict of interest. The authors want to thank Institut Rosell Lallemand for providing the probiotics (L. helveticus R0052 and B. longum R0175) and the vehicle. K. G., J. A.-B., F. F. M., A. B. and T. A. T. contributed to the experiments, data analysis and writing, and R. G. and G. R. contributed to the conception of the experiments, data analysis and writing. 


\section{References}

1. Guck TP, Kavan MG, Elsasser GN, et al. (2001) Assessment and treatment of depression following myocardial infarction. Am Fam Physician 64, 641-648.

2. Meneses R, Almeida MC, Abecasis J, et al. (2007) Depression in patients with myocardial infarction. Rev Port Cardiol 26, $1143-1165$.

3. Frasure-Smith N, Lespérance F \& Talajic M (1993) Depression following myocardial infarction. Impact on 6-month survival. JAMA 270, 1819-1825.

4. Li J, Kuk AY \& Rush AJ (2012) A practical approach to the early identification of antidepressant medication nonresponders. Psychol Med 42, 309-316.

5. Boessen R, Groenwold RH, Knol MJ, et al. (2012) Classifying responders and non-responders; does it help when there is evidence of differentially responding patient groups? $J$ Psychiatr Res 46, 1169-1173.

6. Naumenko SE, Latysheva TV \& Gilinskii MA (2010) Inhibition of norepinephrine reuptake and size of myocardial infarction during focal ischemia and after preconditioning. Bull Exp Biol Med 150, 15-17.

7. Krishnan V \& Nestler EJ (2008) The molecular neurobiology of depression. Nature 455, 894-902.

8. Kaloustian S, Bah TM, Rondeau I, et al. (2009) Tumor necrosis factor-alpha participates in apoptosis in the limbic system after myocardial infarction. Apoptosis 14, 1308-1316.

9. Wann BP, Boucher M, Kaloustian S, et al. (2006) Apoptosis detected in the amygdala following myocardial infarction in the rat. Biol Psychiatry 59, 430-433.

10. Simen BB, Duman CH, Simen AA, et al. (2006) TNFalpha signaling in depression and anxiety: behavioral consequences of individual receptor targeting. Biol Psychiatry 59, 775-785.

11. Raison CL, Capuron L \& Miller AH (2006) Cytokines sing the blues: inflammation and the pathogenesis of depression. Trends Immunol 27, 24-31.

12. Drzyzga LR, Marcinowska A \& Obuchowicz E (2009) Antiapoptotic and neurotrophic effects of antidepressants: a review of clinical and experimental studies. Brain Res Bull 79, 248-257.

13. Rondeau I, Picard S, Bah TM, et al. (2011) Effects of different dietary omega- $6 / 3$ polyunsaturated fatty acids ratios on infarct size and the limbic system after myocardial infarction. Can J Physiol Pharmacol 89, 169-176.

14. Diaz Encarnacion MM, Warner GM, Cheng J, et al. (2011) n-3 Fatty acids block TNF-alpha-stimulated MCP-1 expression in rat mesangial cells. Am J Physiol Renal Physiol 300, F1142-F1151.

15. Wang S, Wu D, Matthan NR, et al. (2009) Reduction in dietary omega-6 polyunsaturated fatty acids: eicosapentaenoic acid plus docosahexaenoic acid ratio minimizes atherosclerotic lesion formation and inflammatory response in the LDL receptor null mouse. Atherosclerosis 204, 147-155.

16. Cazzola M, Tompkins TA \& Matera MG (2010) Immunomodulatory impact of a synbiotic in $\mathrm{T}(\mathrm{h}) 1$ and $\mathrm{T}(\mathrm{h}) 2$ models of infection. Ther Adv Respir Dis 4, 259-270.

17. Wagar LE, Champagne CP, Buckley ND, et al. (2009) Immunomodulatory properties of fermented soy and dairy milks prepared with lactic acid bacteria. J Food Sci $\mathbf{7 4}$, M423-M430.

18. Girard SA, Bah TM, Kaloustian S, et al. (2009) Lactobacillus belveticus and Bifidobacterium longum taken in combination reduce the apoptosis propensity in the limbic system after myocardial infarction in a rat model. Br J Nutr 102, 1420-1425.
19. Boucher M, Wann BP, Kaloustian S, et al. (2006) Reduction of apoptosis in the amygdala by an A2A adenosine receptor agonist following myocardial infarction. Apoptosis 11, 1067-1074.

20. Field A (2009) Discovering Statistics Using SPSS, 3rd ed., pp. 821. London: SAGE Publications Ltd.

21. Siegel S \& Castellan NJJ (1988) Nonparametric Statistics, 2nd ed. Boston, MA: Mcgraw-Hill.

22. Boucher M, Pesant S, Falcao S, et al. (2004) Post-ischemic cardioprotection by $\mathrm{A} 2 \mathrm{~A}$ adenosine receptors: dependent of phosphatidylinositol 3-kinase pathway. I Cardiovasc Pharmacol 43, 416-422.

23. Kaloustian S, Wann BP, Bah TM, et al. (2008) Apoptosis time course in the limbic system after myocardial infarction in the rat. Brain Res 1216, 87-91.

24. Wann BP, Bah TM, Kaloustian S, et al. (2009) Behavioural signs of depression and apoptosis in the limbic system following myocardial infarction: effects of sertraline. J Psychopharmacol 23, 451-459.

25. Jacobson MD, Weil M \& Raff MC (1997) Programmed cell death in animal development. Cell 88, 347-354.

26. Bahrami B, Macfarlane S \& Macfarlane GT (2011) Induction of cytokine formation by human intestinal bacteria in gut epithelial cell lines. J Appl Microbiol 110, 353-363.

27. Hoppu U, Isolauri E, Laakso P, et al. (2012) Probiotics and dietary counselling targeting maternal dietary fat intake modifies breast milk fatty acids and cytokines. Eur J Nutr 51, 211-219.

28. Lyons A, Downer EJ, Crotty S, et al. (2007) CD200 ligand receptor interaction modulates microglial activation in vivo and in vitro: a role for IL-4. J Neurosci 27, 8309-8313.

29. Andoh A, Fujiyama Y, Sumiyoshi K, et al. (1996) Interleukin 4 acts as an inducer of decay-accelerating factor gene expression in human intestinal epithelial cells. Gastroenterology 111, 911-918.

30. Arita M, Ohira T, Sun YP, et al. (2007) Resolvin E1 selectively interacts with leukotriene B4 receptor BLT1 and ChemR23 to regulate inflammation. J Immunol 178, 3912-3917.

31. Krishnamoorthy S, Recchiuti A, Chiang N, et al. (2010) Resolvin D1 binds human phagocytes with evidence for proresolving receptors. Proc Natl Acad Sci US A 107, 1660-1665.

32. Adkins Y \& Kelley DS (2010) Mechanisms underlying the cardioprotective effects of omega-3 polyunsaturated fatty acids. J Nutr Biochem 21, 781-792.

33. Ross JA, Maingay JP, Fearon KC, et al. (2003) Eicosapentaenoic acid perturbs signalling via the NFkappaB transcriptional pathway in pancreatic tumour cells. Int J Oncol 23, 1733-1738.

34. Mukherjee PK, Marcheselli VL, Barreiro S, et al. (2007) Neurotrophins enhance retinal pigment epithelial cell survival through neuroprotectin D1 signaling. Proc Natl Acad Sci U S A 104, 13152-13157.

35. Belayev L, Khoutorova L, Atkins KD, et al. (2009) Robust docosahexaenoic acid-mediated neuroprotection in a rat model of transient, focal cerebral ischemia. Stroke $\mathbf{4 0}$, 3121-3126.

36. Zhu BQ, Sievers RE, Sun YP, et al. (1994) Is the reduction of myocardial infarct size by dietary fish oil the result of altered platelet function? Am Heart J 127, 744-755.

37. Heller A, Stehr S \& Koch T (2006) Omega 3 Fatty Acids in Clinical Nutrition, 1st ed. New York, NY: Nova Science Publishers, Inc.

38. Lands WEM (2005) Fish, Omega-3 and Human Health, 2nd ed. Champaign, IL: American Oil Chemists' Society Press. 